\title{
Separation of antibody drug conjugate species by RPLC: a generic method
} development approach

\author{
AUTHORS: Szabolcs FEKETE ${ }^{1 *}$, Imre MOLNÁR ${ }^{2}$, Davy GUILLARME ${ }^{1}$ \\ ${ }^{1}$ School of Pharmaceutical Sciences, University of Geneva, University of Lausanne, CMU - \\ Rue Michel Servet, 1, 1211 Geneva 4 - Switzerland \\ ${ }^{2}$ Molnár-Institute for Applied Chromatography, Schneegloeckchen strasse 47, 10407 Berlin, \\ Germany
}

CORRESPONDENCE: Szabolcs FEKETE

Phone: +412237963 34

Fax: +412237968 08

E-mail: szabolcs.fekete@unige.ch 
${ }^{1}$ School of Pharmaceutical Sciences, University of Geneva, University of Lausanne, CMU - Rue Michel Servet, 1, 71211 Geneva 4 - Switzerland

$8{ }^{2}$ Molnár-Institute for Applied Chromatography, Schneegloeckchen strasse 47, 10407 Berlin, Germany

ABSTRACT

11 This study reports the use of modelling software for the successful method development of 12 IgG1 cysteine conjugated antibody drug conjugate (ADC) in RPLC. The goal of such a 13 method is to be able to calculate the average drug to antibody ratio (DAR) of and ADC 14 product. A generic method development strategy was proposed including the optimization of mobile phase temperature, gradient profile and mobile phase ternary composition. For the first time, a 3D retention modelling was presented for large therapeutic protein. Based on a

17 limited number of preliminary experiments, a fast and efficient separation of the DAR species 18 of a commercial ADC sample, namely brentuximab vedotin, was achieved. The prediction 19 offered by the retention model was found to be highly reliable, with an average error of 20 retention time prediction always lower than $0.5 \%$ using a $2 \mathrm{D}$ or $3 \mathrm{D}$ retention models. For 21 routine purpose, four to six initial experiments were required to build the $2 \mathrm{D}$ retention models, while 12 experiments were recommended to create the 3D model. At the end, RPLC can

23 therefore be considered as a good method for estimating the average DAR of an ADC, 24 based on the observed peak area ratios of RPLC chromatogram of the reduced ADC 25 sample. 
Keywords:

29 Retention modeling, antibody drug conjugate, brentuximab vedotin, method development, 30 DryLab, reversed phase liquid chromatography

\section{INTRODUCTION}

33 Antibody drug conjugates (ADCs) are chemotherapeutics constituted of a cytotoxic chemical

34 drug linked covalently - via peptide linker - to a monoclonal antibody (mAb) [1]. They are used for the treatment of cancer by combining the proven antigen-specific selectivity and activity of mAbs with the potency of highly cytotoxic small molecules [2]. Drug conjugation can be achieved via reactions at different amino acid residues such as at lysine side chains amines (lysine conjugation), at cysteine thiol groups after reduction of the interchain disulfide bonds (cysteine conjugation), or at engineered cysteine residues at specific sites on a mAb $[3,4]$. The addition of the drugs results in a heterogeneous population of ADCs that differ in the number of drugs per antibody, and this mixture can be described by an average drug to antibody ratio (DAR) [4]. Structurally, immunoglobulin G1 (IgG1) mAbs contain four interchain disulphide bridges. Among those, two are located in the hinge region and connect the two heavy chains $(H)$ while the other two connect the $H$ and light chain $(L)$. Therefore, a cysteine linked IgG1 ADC is composed of a heterogeneous mixture of $0,2,4,6$ or 8 drug conjugations [5]. An odd number of conjugated drugs on an ADC is typical indicative of 47 degradation [6,7]. In this study, we focus only on a commercially available cysteine linked IgG1 ADC, namely brentuximab vedotin.

One of the most important critical quality attributes (CQA) of an ADC is the average DAR, since it determines the amount of "payload" that can be delivered to a tumor cell and can

51 affect efficacy and safety [3]. A variety of analytical methods have been used to measure the 52 average DAR, including UV-VIS spectroscopic, liquid chromatographic, and native mass 53 spectrometric methods [4,8-19]. Among liquid chromatographic methods, hydrophobic 54 interaction chromatography (HIC) and reversed phase chromatography (RPLC) are routinely used. The former approach separates the intact DAR species under non-denaturating 
conditions, while RPLC is mostly used to separate the DAR species of reduced ADC sample related to the $\mathrm{H}$ or $\mathrm{L}$ chains as $\mathrm{L} 0, \mathrm{~L} 1$ and $H 0, H 1, H 2$ and $H 3$ species.

58 In addition to information about the average DAR, multiple methods have been applied to analyze the distribution of drug linked forms and their positional isomers. This is again an important characteristic because different forms may have different pharmacokinetic and

61 toxicological properties [3,16]. A number of methods for this task are based on the use of mass spectrometry (MS) [20-23]. The separation of intact DARs in HIC also allows the isolation of pure species and permits further characterization (e.g. by capillary electrophoresis-sodium dodecyl sulphate and capillary iso-electric focusing (CE-SDS, cIEF) or cell based bioassay) $[3,5]$. The complete characterization of ADC DAR positional isomers can also be performed on-line, by peptide mapping LC-MS or two dimensional (HIC $x$ RPLC)-MS analysis of the native $\operatorname{ADC}[6,7,24,25]$. In addition to average DAR, DAR distribution and positional isomer analysis, a number of complementary methods can be applied for further characterization of ADCs to determine residual free drugs, size variants or charge variants [25].

As mentioned earlier, RPLC is mostly used to separate the DAR species of reduced ADC related to $H$ and $L$, but can also be applied on the native $A D C$ to separate $L, H, H H, H L$ and $\mathrm{HHL}$ fragments. The latter one can be useful, as a second dimension separation prior to MS. In the literature, only "historical" methods are reported, using a mobile phase containing 0.1 $\%$ trifluoroacetic-acid (TFA), an acetonitrile gradient and elevated temperature of $70-80{ }^{\circ} \mathrm{C}$. However, nothing is reported about systematic method development, optimization procedure or retention behavior. In this study, we offer a possible generic method development approach for lgG1 cysteine conjugations. Both 2D and 3D retention models are discussed. The impact of temperature and mobile phase ternary composition was also studied. Finally,

80 four, six and twelve experiments based designs were suggested for routine purposes. This 81 new approach allows a fast and accurate systematic method development for the efficient separation of ADC DAR species and fragments. 


\section{2. Experimental}

$85 \quad 2.1$ Chemicals and columns

86 Water was obtained from a Milli-Q Purification System from Millipore (Bedford, MA, USA).

87 Acetonitrile $(\mathrm{ACN})$ and methanol $(\mathrm{MeOH})$ were ULC-MS grade and purchased from Biosolve 88 (Valkenswaald, Netherlands). Dithiothreitol (DTT, $\geq 99.0 \%$ ) and trifluoroacetic acid (TFA, 89 99\%) were purchased from Sigma-Aldrich (Buchs, Switzerland).

90 Brentuximab vedotin as therapeutic monoclonal antibody drug conjugate ( $\lg G 1$ cysteine 91 linked $A D C$ ) was kindly provided by the Center of Immunology Pierre Fabre (Saint-Julien en 92 Genevois, France).

93 The AdvanceBio RP-mAb C4 (3.5 $\mu \mathrm{m}, 150 \mathrm{~mm} \times 2.1 \mathrm{~mm}, 450 \AA$ A $)$ column was purchased 94 from Agilent (USA).

95

2.2 Equipment and software

Measurements were performed using a Waters Acquity UPLC ${ }^{\text {TM }}$ I-Class system equipped with a binary solvent delivery pump (maximum flow rate of $2 \mathrm{~mL} / \mathrm{min}$ and upper pressure limit of 1200 bar), an auto-sampler and UV detector. The system includes a flow through needle (FTN) injection system with $15 \mu \mathrm{L}$ needle and a $0.5 \mu \mathrm{L}$ UV flow-cell. The overall extra-column volume $\left(V_{\text {ext }}\right)$ was about $7.5 \mu \mathrm{L}$ as measured from the injection seat of the auto-sampler to the detector cell. The dwell volume was measured as $V_{d}=0.09 \mathrm{~mL}$.

103 Data acquisition and instrument control were performed by Empower Pro 3 software 104 (Waters). Calculations and data transferring were achieved with Excel (Microsoft). Retention and resolution modelling was performed with DryLab ${ }^{\circledR} 4$ software (Molnár-Institute, Berlin, Germany).

2.3. Mobile phase composition and sample preparation

109 For the gradient separation of ADC DARs and its fragments, the mobile phase " $A$ " consisted 110 of $0.1 \%$ TFA in water, whereas the mobile phase "B" was $0.1 \%$ TFA in organic solvent 111 having different possible compositions of acetonitrile and methanol. 
112 Native brentuximab vedotin was injected as received $(5 \mathrm{mg} / \mathrm{mL})$ using low volume insert 113 vials.

114 Reduced brentuximab vedotin sample was prepared by the addition of DTT solution (10 mM) 115 and incubation at $30{ }^{\circ} \mathrm{C}$ for $60 \mathrm{~min}$. The $\mathrm{ADC}$ was completely converted into the light and 116 heavy chain components. Then, sample was injected from low volume insert vial.

118 2.4. Investigation of retention properties of ADC species (preliminary studies)

119 Native brentuximab vedotin was eluted in RPLC mode using linear gradients. The effect of 120 the gradient profile, temperature and organic modifier on the retention and selectivity of ADC 121 species was studied. Detection was carried out at $280 \mathrm{~nm}$, and $1 \mu \mathrm{L}$ sample was injected.

122 First, the effect of gradient steepness (gradient time, $t_{G}$ ) on retention and selectivity was 123 evaluated. A generic linear gradient, starting from $25 \%$ to $50 \%$ B was applied at a flow rate of $1240.3 \mathrm{~mL} / \mathrm{min}$. For mobile phase $\mathrm{B}$, acetonitrile was used as organic modifier and the 125 experiments were performed at $\mathrm{T}=90{ }^{\circ} \mathrm{C}$. In our previous study, the validity of linear solvent 126 strength model (LSS) was proved for mAbs and subunits in the practically relevant design 127 space (DS) [26]. The gradient time was varied as $t_{G}=6,12$ and $18 \mathrm{~min}$. The observed 128 apparent (or gradient) retention factors $\left(k_{\text {app }}\right)$ were plotted against $t_{G}$ and the change in 129 selectivity (elution order) was followed.

130 Based on previous experiments, high temperature seemed to be mandatory for correct peak 131 shape and recovery [27]. Therefore, all the experiments were performed at mobile phase 132 temperature of $\mathrm{T} \geq 70{ }^{\circ} \mathrm{C}$. Short gradient runs $\left(t_{G}=6 \mathrm{~min}\right)$ were carried out at $70,75,80,85$ 133 and $90{ }^{\circ} \mathrm{C}$ (up to the upper temperature limit of the column). Our purpose was to illustrate the 134 effect of temperature on the retention and recovery. Plots of log $k_{a p p} v s .1 / T$ and recovery vs. 135 T were made. For the estimation of recovery, peak area values were normalized to the ones 136 observed at the highest temperature $\left(90^{\circ} \mathrm{C}\right)$.

137 At last, the impact of the organic modifier was studied by running gradients with mixtures of 138 acetonitrile and methanol. Aprotic and protic solvents may have an impact on the solvation of 139 ADC species and can interact in different ways with the proteins and also with the residual 
140 acidic silanols of the silica based stationary phase. Therefore, differences in retention and 141 selectivity were expected. Ternary mobile phases were prepared using water, acetonitrile 142 and methanol. The ternary composition $(t C)$ of mobile phase $B$ was set as $0,20,40$ and $50 \%$ $143 \mathrm{MeOH}$ in $\mathrm{ACN}$. Since $\mathrm{MeOH}$ has a lower eluent strength than $\mathrm{ACN}$, the gradients were run in 144 an extended $\% \mathrm{~B}$ range $(30-80 \% \mathrm{~B})$, to elute all the species with all mobile phase 145 compositions. The experiments were performed at $\mathrm{T}=90{ }^{\circ} \mathrm{C}$, and $t_{G}=6 \mathrm{~min}$. Higher than $14650 \% \mathrm{MeOH}$ content resulted in unacceptable recovery and too high retention.

\subsection{Systematic method optimization}

Snyder, Dolan and co-workers recommended initial basic runs for multifactorial experimental designs already in the 90's [28]. A general approach is to simultaneously model the effect of temperature and gradient steepness $\left(t_{G}\right)$ on selectivity with a previously selected RPLC column $[29,30]$. Then, with the help of resolution maps generated by modelling software which show the critical resolution of the peaks to be separated [31] - the gradient program and column temperature can be rapidly and efficiently optimized. Today - thanks to new developments of modelling software - it is also possible to simultaneously optimize three method variables. Beside temperature and gradient steepness, ternary mobile phase composition or mobile phase $\mathrm{pH}$ are often selected as third variable [32-34]. Since neither

158 ternary composition nor mobile phase $\mathrm{pH}$ impact retention in a linear fashion, their effect 159 have to be measured at least at three levels. The dependence of retention on both $t_{G}$ and $\mathrm{T}$ 160 can be transferred to linear relationships and therefore necessitate only two experiments for 161 one variable to build up an accurate retention model. In our DS, these linear relationships were found to be valid for large ADC species.

163 In this study, the most information retention model for ADC subunits was found to be a

164 twelve experiments based combined 3D model of $t_{G} \times T \times t C$. The gradient time was set as $t_{G 1}$ $165=10 \mathrm{~min}$ and $t_{\mathrm{G} 2}=20 \mathrm{~min}$, temperature was studied at $\mathrm{T}_{1}=75^{\circ} \mathrm{C}$ and at $\mathrm{T}_{2}=90{ }^{\circ} \mathrm{C}$ while 166 ternary composition was set at $t C_{1}=0 \%, t C_{2}=10 \%$ and $t C_{3}=20 \% \mathrm{MeOH}$ in $\mathrm{ACN}$. Longer 167 gradients than 20 min were not considered to avoid on column degradation of large ADC 
168 species at elevated temperature. The experiments were performed at a flow rate of 0.3 $169 \mathrm{~mL} / \mathrm{min}$ (25 to $50 \% \mathrm{~B}$, linear gradients). The accuracy of retention models were verified in 170 the centre point of the DS, namely $t_{G}=15 \mathrm{~min}, \mathrm{~T}=82,5 \mathrm{C}$ and $t C=10 \% \mathrm{MeOH}$.

171 Mobile phase $\mathrm{pH}$ was not an important method variable, and $0.1 \%$ TFA is commonly added

172 to the mobile phase to have ion-pairs with the positively charged proteins and a $\mathrm{pH}$ of around 1732 in the mobile phase.

174 Simplified 2D retention models were also suggested as $t_{G} \times T$ and $t_{G} \times t C$. The former one 175 requires four initial experiments, while the latter one needs six experimental runs. These 2D 176 models were built up on the basis of experimental runs performed for the 3D model. For the 177 first $2 \mathrm{D}$ model $t_{G 1}=10 \mathrm{~min}, t_{G 2}=20 \mathrm{~min}, \mathrm{~T}_{1}=75{ }^{\circ} \mathrm{C}$ and $\mathrm{T}_{2}=90{ }^{\circ} \mathrm{C}$ were considered (with $0 \%$ $178 \mathrm{MeOH}$ in mobile phase $\mathrm{B}$ ). For the second $2 \mathrm{D}$ model $t_{G 1}=10 \mathrm{~min}, t_{\mathrm{G} 2}=20 \mathrm{~min}, t C_{1}=0 \%, t C_{2}$ $179=10 \%$ and $t C_{3}=20 \% \mathrm{MeOH}$ in $\mathrm{ACN}$ were considered at $\mathrm{T}=90{ }^{\circ} \mathrm{C}$.

180 Optimal conditions (working points) were determined for both the native and reduced 181 brentuximab vedotin and then the accuracy of retention time prediction was studied. 182 Simulated robustness test was also performed around the working point.

183

\section{Results and Discussion}

3.1. Preliminary measurements

186 In RPLC, interaction with the stationary phase is mediated predominantly through 187 hydrophobic interactions between the nonpolar amino acid residues of proteins and the 188 immobilized n-alkyl ligands. Solutes are eluted in their order of increasing molecular 189 hydrophobicity. The retention of $\mathrm{mAb}$ and $\mathrm{ADC}$ species, i.e. large biomolecules is strongly 190 dependent on small changes in the solvent strength and a very small change $(<1 \%)$ in the 191 organic modifier content could lead to a significant shift in the retention. For this reason, 192 isocratic conditions are impractical, and gradient elution is mandatory. In addition, the peak 193 shape of large molecules is always better in gradient mode compared to isocratic one due to 194 band focusing effects. The LSS model is the widely accepted theory which describes the 195 analyte retention as a function of the volume fraction $(\Phi)$ of the eluting B solvent. This model 
generally well describes the retention of numerous types of analytes including large proteins

197 such as mAbs or mAb sub-units [26].

198 Figure 1 shows the impact of $t_{G}$ on selectivity and $k_{a p p}$. Interestingly, the selectivity between some peaks changes drastically with $t_{G}$. With a fast gradient $\left(t_{G}=6 \mathrm{~min}\right)$, the first three peaks 200 elute in the following order: $\mathrm{A}, \mathrm{B}$ and $\mathrm{C}$. When increasing $t_{G}$ to $12 \mathrm{~min}$, the elution order of $\mathrm{B}$ 201 and $\mathrm{C}$ changes. Further increasing $t_{G}(18 \mathrm{~min})$ results in a new elution order, namely $\mathrm{C}, \mathrm{A}$ 202 and $\mathrm{B}$. Figure $1 \mathrm{D}$ well represents the effect of $t_{G}$ on selectivity since the fitted curves of $\mathrm{k}_{\text {app }}$ 203 vs $t_{G}$ for peaks $\mathrm{A}, \mathrm{B}$ and $\mathrm{C}$ cross each other in the studied range. Moreover, the LSS 204 behavior of the ADC species was also illustrated, since linear trends fit perfectly with the experimental data. These preliminary results suggest that gradient steepness is a very important method variable to optimize ADC separations under RPLC conditions.

207 The effect of temperature on retention factor is generally expressed by the van't Hoff 208 relationship. When log $k_{\text {app }}$ is plotted against $1 / \mathrm{T}$, a linear relationship is expected for regular 209 solutes. However, with large biomolecules, the effect of temperature on retention becomes 210 more complex. Depending on the stability of the secondary structure, the molecules unfold to 211 various extents and hence interact with the stationary phase with various strengths [35]. Due 212 to the different conformation-dependent responses of proteins at elevated temperatures, the 213 change in retention can be very different $[36,37]$. Another issue related to temperature is the 214 possible thermal degradation of proteins when working at elevated temperature [27]. It was 215 shown for mAbs that analysis time should be kept below 20 min to avoid degradation at $70-$ $21690{ }^{\circ} \mathrm{C}$. On the other hand, high temperature is mandatory to limit protein adsorption on the 217 stationary phase. A temperature lower than $70{ }^{\circ} \mathrm{C}$ results in unacceptable recovery for most $218 \mathrm{mAbs}$ [38]. Therefore, it is important to work in a limited range in terms of temperature and 219 analysis time. If the analysis time is no longer than 20 min, then, a van't Hoff like behavior is 220 expected between 70 and $90{ }^{\circ} \mathrm{C}$ for mAbs and subunits [26]. Figure $2 \mathrm{~A}$ shows the observed 221 chromatograms of brentuximab vedotin measured at $70,75,80,85$ and $90{ }^{\circ} \mathrm{C}$. As shown in 222 figure $2 \mathrm{~A}$, the ratio of peak areas of the main peaks $(A-F)$ changes with temperature. The 223 change of peak areas was especially important for peaks A, B and D. As reported in Figure 2 
$224 \mathrm{~B}$, only 27,47 and $59 \%$ of peak areas was observed at $70{ }^{\circ} \mathrm{C}$ for peaks $\mathrm{A}$, B and D 225 compared to $90{ }^{\circ} \mathrm{C}$. Based on previous studies [6,7], these peaks probably correspond to the 226 naked $\mathrm{mAb}$ (and mAb with two drugs on the $\mathrm{H}$ in the hinge region), the conjugated light chain 227 (L1) and different $\mathrm{HHL}$ species (HHL1 and HHL3). To have at least $50 \%$ recovery for all 228 peaks, the temperature should not be set below $75^{\circ} \mathrm{C}$. Figure $2 \mathrm{C}$ confirms the van't Hoff like 229 behavior of these ADC species within the applied temperature range. It is important since it 230 enables to study the effect of temperature on retention only at two levels and simplifies the 231 experiments.

232 Another interesting variable is the mobile phase ternary composition. Figure 3 shows the 233 impact of organic modifier nature in mobile phase B. Increasing $\mathrm{MeOH}$ vs. ACN results in 234 expected increase of retention. In addition, some differences in selectivity were also 235 observed. However, a drawback of using huge $\mathrm{MeOH}$ fraction is the non-desired adsorption 236 of the ADC species onto the stationary phase. The sum of peak areas decreases when 237 increasing the $\mathrm{MeOH}$ content. At $50 \% \mathrm{MeOH}$, only about $30 \%$ of total peak area was 238 observed, which is not acceptable. The $\mathrm{MeOH}$ fraction of mobile phase $\mathrm{B}$ should then, not be 239 larger than $20 \%$ to get appropriate recovery for all peaks.

240 To conclude on the preliminary studies, $t_{G}, \mathrm{~T}$, and $t C$ all seem to be interesting variables to 241 optimize or adjust the separation of ADC species in RPLC. However, these 3 parameters can 242 only be tuned in a limited design space, when considering possible recovery and degradation 243 issues.

\subsection{D experimental design and retention model}

246 Based on the preliminary studies, a 3D design ( $t_{G} \times T \times t C$ model) was suggested. The levels 247 (and values) of such an experimental design were illustrated on Figure 4 for a $150 \times 2.1 \mathrm{~mm}$ 248 column operating at $0.3 \mathrm{~mL} / \mathrm{min}$. Several additional preliminary experiments were performed 249 to determine the values of $t_{G}$ for the initial runs ranging between 4 and $20 \mathrm{~min}$. For most 250 solutes (including proteins), a factor of three was used between the two set levels of $t_{G}$, to 251 provide accurate retention modelling (e.g. $t_{G 1}=6 \mathrm{~min}$ and $t_{G 2}=18 \mathrm{~min}$ ). However, with the 
252 ADC subunits analysed in the present study, combining any shorter gradients than $10 \mathrm{~min}$ 253 with a longer one $\left(t_{G}>15 \mathrm{~min}\right)$ resulted in inaccurate retention model. This is probably due to 254 the very high slope of the LSS model $(S)$ for these large proteins. Finally, it was found that 255 performing $t_{G 1}=10 \mathrm{~min}$ and $t_{G 2}=20$ min gradients (difference of a factor two) results in 256 accurate retention modelling and enables the precise prediction of retention times for any 257 gradient program (linear and multilinear too and for extrapolated $t_{G}$ such as $t_{G}<10 \mathrm{~min}$ ).

258 The experimental data from the twelve experiments were entered into the modelling 259 software. After processing and checking the data accuracy, the retention times of 10 peaks 260 from native ADC and 14 peaks of reduced ADC were matched in each of the chromatograms 261 by using the PeakMatch module of the DryLab software. The peak tracking process was based on peak areas. All the data were automatically transferred into the modelling software

263 but small adjustments for the peak widths were required to get realistic peak capacity in the 264 simulated chromatograms [39]. Please note, that peak tracking based on peak area was not 265 obvious in this work, due to the fact that the sum of the peak areas was expectedly lower at $26675^{\circ} \mathrm{C}$ vs. $90{ }^{\circ} \mathrm{C}$ because of the significant on-column adsorption at lower temperature. Some 267 ADC subunits adsorb more intensively onto the stationary phase, while for other peaks (e.g. 268 H3 or L0), the adsorption was less critical. Therefore, peak movements have to be followed 269 and understood before matching the peak areas. Manual adjustment may have to be 270 performed.

271 After building up the retention model, its accuracy was experimentally verified. Both native 272 and reduced ADC samples were run in the centre point of the experimental design. Retention 273 times and chromatograms were also predicted for this condition. Figure 5 shows the 274 predicted and measured chromatograms and the identification of the 10 and 14 peaks 275 included in the models. As shown in figure 5, the experimentally observed and predicted 276 chromatograms were in very good agreement. Tables 1 and 2 present the difference and \% 277 error of measured and calculated retention times for the native and reduced ADC, 278 respectively. There was no more than $0.5 \%$ error, and the average error of retention time 279 prediction was below $0.3 \%$. 
280 The verification of the model was assessed by creating resolution maps (Figure 6). The 281 colour code in these resolution maps represents the value of the critical resolution (Rs), with 282 warm "red" colours corresponding to large resolution values (Rs > 1.0) and cold "blue" 283 colours correspond to low resolution values (Rs $<0.3)$. The visual inspection of the cubes 284 show the largest red region, where the method is probably robust and the resolutions of all 285 peaks in the chromatogram are the best that can be achieved (when using the initial linear 286 gradient). Based on the resolution cubes, the starting point of the optimization can easily be 287 selected. Further optimization can be done by changing the $\mathrm{B} \%$ of initial and final mobile 288 phase composition. After changing the $\mathrm{B} \%$, again the effect of temperature and ternary 289 composition worth re-studying. After further optimization, a gradient from 29 to $42 \% \mathrm{~B}$, with $t_{G}$ $290=14 \min$ at $\mathrm{T}=90{ }^{\circ} \mathrm{C}$ and $t C=0 \% \mathrm{MeOH}$ gave a fast and appropriate separation for the 291 native ADC sample (Figure 7). The experimental verification of this working point again 292 showed high prediction accuracy. The average error of retention time prediction was lower 293 than $0.5 \%$. For the reduced ADC, the optimal conditions were found as a gradient of $31-48$ $294 \% \mathrm{~B}, t_{G}=18 \mathrm{~min}, \mathrm{~T}=90{ }^{\circ} \mathrm{C}$ and $t \mathrm{C}=20 \% \mathrm{MeOH}$ (Figure 8). Similarly, the predicted and 295 experimental chromatograms were in good agreement (lower than $0.5 \%$ error).

296 As illustrated by this example, this generic 3D retention model and optimization for cysteine 297 linked ADCs seems to be interesting. It can also be useful for laboratories working under 298 regulated conditions, since all the possible combinations of method variables can quickly be 299 checked.

300 The time required for this 12 runs based design and its verification is about 7-8 hours for one 301 sample and assuming duplicate injections $(2 \times(6 \times 10 \mathrm{~min}+6 \times 20 \mathrm{~min}+1 \times 15 \mathrm{~min})+$ system 302 equilibration). Then the understanding of peak movements, peak tracking, importing 303 chromatograms and creating the model take around 5-6 hours. Finally, the optimization and 304 then the experimental verification of the selected working point take an additional 2-3 hours 305 of work. In total, this optimization approach of ADC species separations in RPLC mode 306 requires 2-3 working days. 


\subsection{Simulated robustness testing}

309 An interesting feature of DryLab 4 modelling software is its ability to perform an in-depth 310 "modelled" robustness testing [34]. From the design space, as defined in a resolution cube, it 311 is possible to get robustness information for the measured variables, including $t_{G}, T$ and $t C$. 312 The influence of additional parameters, such as flow rate or initial- and final-\%B of the 313 gradient, on retention time can be predicted for all compounds. Consequently, the impact of 314 changes in any of these 6 variables/parameters on the resolution can be assessed using a 315 simulated $2^{6}$ or $3^{6}$ type factorial design. After defining the possible deviations from the 316 nominal values, 64 or 729 experiments can be virtually performed. At the end, the software 317 provides a 'frequency distribution graph' showing how often $(\mathrm{N})$ a certain critical resolution 318 occurs under any combination of possible parameters. On the other hand, 'regression 319 coefficients' can also be obtained to show the effect of each parameter, related to the 320 selected deviation from the nominal value, for the critical resolution.

321 This simulated robustness test was performed around a selected working point as $t_{G}=27 \pm$ $3220.5 \mathrm{~min}, \mathrm{~T}=87 \pm 1{ }^{\circ} \mathrm{C}, t \mathrm{C}=5 \pm 1 \% \mathrm{MeOH}$, flow rate: $0.30 \pm 0.05 \mathrm{~mL} / \mathrm{min}$, initial \%B: $25 \pm 1$, 323 final \%B: $50 \pm 1$ ). This condition allowed the best separation of the $L 1$ and $H 0$ peaks (as critical peak pairs) of the reduced brentuximab vedotin (Figure 9). The baseline separation of

325 the $\mathrm{LO}, \mathrm{L} 1, \mathrm{H} 0, \mathrm{H} 1, \mathrm{H} 2$ and $\mathrm{H} 3$ species are important for the determination of average DAR. 326 The separation of positional isomers of a given species (e.g. $\mathrm{H} 1$ or $\mathrm{H} 2$ have 3 different 327 positional isomers) is not mandatory for this purpose. Please note, that the total run time is $32827 \mathrm{~min}$ but the retention time of the last eluting peak is below $20 \mathrm{~min}$, thus possible thermal 329 degradation is not an issue. Performing the 729 virtual experiments showed that all the 330 experiments met the Rs $>1.5$ criterion, thus the conditions defined by our selected working 331 point can be considered as robust (Figure $9 \mathrm{C}$ ). The lowest observed resolution was Rs = 3321.73 which is still acceptable. The gradient time was found to be the most influential method 333 variable.

334 Another important fact is that the separation on figure 9 takes only 27 min in total while 335 previously a 70 min long RP method was reported for the same sample in ref [5]. The gain in 
analysis time (3 times faster separation) and better separation quality (higher resolution

337 between positional isomers) here is due to the systematic optimization and the 338 understanding of retention behavior.

\subsection{Simplified $2 \mathrm{D}$ experimental designs}

341 Obviously, the 3D retention model can be simplified to 2D models if required (e.g. to gain in 342 time or if $3 \mathrm{D}$ retention modeling software is not available). One possibility is to select a $t_{G} \times T$ 343 model which requires four initial runs, while the other choice is to perform a $t_{G} \mathrm{X} t C$ model 344 which necessitates six experiments (Figure 10).

345 To perform a $t_{G} \times T$ model, ternary mobile phase composition is not suggested. Since the 346 best recovery was obtained with ACN, the mobile phase B should preferably be $0.1 \%$ TFA in 347 ACN as a first choice. The time required for the experiments is only around 2-3 hours ( $2 \times(2$ $348 \times 10 \mathrm{~min}+2 \times 20 \mathrm{~min}$ ) + system equilibration) for one sample (with duplicate injections). 349 Figure 11 shows the obtained 2D resolution maps for the native and reduced ADC. The blue 350 lines indicate co-elutions (and therefore elution order changes). Since the blue lines oriented 351 in both vertical and horizontal directions on the map, the design space indeed seems to be 352 well selected since both method variables play an important role in the overall quality of the 353 separation.

354 Figure 12 shows the obtained $2 \mathrm{D}$ resolution maps for a $t_{G} \mathrm{X} t C$ model. In this case, the 355 temperature should be set as high as possible to avoid recovery issues (e.g. $\mathrm{T}=90^{\circ} \mathrm{C}$ ). This 356 experimental design takes around $3-4$ hours of work $(2 \times(3 \times 10 \mathrm{~min}+3 \times 20 \mathrm{~min})+$ system 357 equilibration). The maps again suggest that both variable ( $t_{G}$ and $t C$ ) have a huge impact on 358 the critical resolution and therefore makes this model interesting for routine applications.

359 Both 2D models provided similar maximum resolution and analysis time as for an optimal 360 method. If further optimization is required then the $t_{G} \mathrm{X} T$ model can be repeated with a 361 ternary mobile phase (e.g. $20 \% \mathrm{MeOH}+80 \% \mathrm{ACN}$ as organic solvent) while the $t_{G} \mathrm{X} t \mathrm{C}$ 362 model can be performed again, but at a different temperature (e.g. at $80^{\circ} \mathrm{C}$ ). This repeated 
experiments may perform better quality of separation. If it is not the case, then the best

364 choice is to perform one of these 2D models on a different stationary phase.

365

3.5. Determination of the average DAR

367 One method of choice to determine the average DAR of an ADC is based on the observed 368 peak area ratios of RPLC chromatogram of the reduced ADC:

$$
D A R=2 x\left(\sum_{i=0}^{1} \text { weighted peak area of } L(i)+\sum_{i=0}^{3} \text { weighted peak area of } H(i)\right) / 100
$$

369 Both optimum conditions (presented in Figures 8 and 9) resulted in DAR $=4.0$. This value is 370 in good agreement with those reported in the literature $[6,7,17]$. However, it is worth 371 mentioning that the average DAR was calculated for all the initial runs (at 75 and $90{ }^{\circ} \mathrm{C}$ ), and

372 we systematically observed larger DAR value (as DAR $=4.2-4.3$ ) at $75^{\circ} \mathrm{C}$ compared to 90 $373{ }^{\circ} \mathrm{C}(\mathrm{DAR}=4.0)$. Finally, this is not surprising since the poorer recovery of some species 374 results in differences in pear area \% distribution. Logically, the DAR determined at lower 375 temperature is not correct, and therefore attention should be paid when calculating the 376 average DAR from RPLC chromatograms.

\section{Conclusions}

379 A new 3D retention model was suggested and successfully applied in RPLC for IgG1 380 cysteine conjugated antibody drug conjugate (ADC). In total, the optimization of this 3D 381 retention model required only 2-3 working days (12 experiments for one sample). In addition 382 to the $3 \mathrm{D}$ model, simplified 2D models could also be done for fast method development 383 which took less than one day (4 or 6 experiments with one sample). Generic (platform) 384 method development approach was found to be feasible. The time saving of this systematic 385 methodology is obvious compared to trial and error approach.

386 The most important method variables to optimize ADC separation were the gradient 387 steepness (1), mobile phase temperature (2) and mobile phase ternary composition (3). The 
388 retention time prediction offered by the retention models was found to be highly accurate, 389 with an average error lower than $0.5 \%$.

390 The developed RPLC method can be considered for determining the average DAR of an 391 ADC, based on the observed peak area ratios of the reduced ADC sample. The native ADC 392 separation can also be useful for multidimensional separations in the second dimension to

393 separate the positional isomers and different species and enabling the MS identification.

394 It also worth mentioning that - by understanding the retention behavior of ADC peaks 395 analysis time could be shortened into the 20-25 min range while 60-70 min long separations 396 were reported previously.

397

398 5. Acknowledgements

399 The authors wish to thank Alain Beck from Pierre Fabre for providing the antibody drug 400 conjugate sample.

401 Davy Guillarme wishes to thank the Swiss National Science Foundation for support through a 402 fellowship to Szabolcs Fekete (31003A 159494).

403 
405 [1] R.V.J. Chari, Targeted cancer therapy: conferring specificity to cytotoxic drugs, Acc. 406 Chem. Res. 41 (2008) 98-107.

407 [2] S. Ornes, Antribody drug conjugates, Proc. Natl. Acad. Sci. U.S.A. 110 (2013) 13695.

408 [3] A. Wakankar, Y. Chen, Y. Gokarn, F.S. Jacobson, Analytical methods for 409 physicochemical characterization of antibody drug conjugates, mAbs 3 (2011) 161-172.

410 [4] B. Wiggins, L. Liu-Shin, H. Yamaguchi, G. Ratnaswamy, Characterization of cysteine411 linked conjugation profiles of immunoglobulin G1 and immunoglobulin G2 antibody-drug 412 conjugates, Pharm. Drug Deliv. Pharm. Tech. 104 (2015) 1362-1372.

413 [5] L.N. Le, J.M. Moore, J. Ouyang, X. Chen, M.D. Nguyen, W.J. Galush, Profiling antibody 414 drug conjugate positional isomers: A system of-equations approach. Anal Chem 84 (2012) $4157479-7486$.

416 [6] S. Heinisch, M. Sarrut, D. Guillarme, A. Beck, S. Fekete, M.C.J. Bussat, O. Colas, 417 Analysis of antibody-drug conjugates by comprehensive on-line two-dimensional 418 hydrophobic interaction chromatography $x$ reversed phase liquid chromatography 419 hyphenated to high resolution mass spectrometry. II - Identification of sub-units for the 420 characterization of even and odd load drug species, J. Chromatogr. B, 1032 (2016) 91-102.

421 [7] M. Sarrut, A. Corgier, S. Fekete, D. Guillarme, D. Lascoux, M.C.J. Bussat, A. Beck, S. 422 Heinisch, Analysis of antibody-drug conjugates by comprehensive on-line two-dimensional 423 hydrophobic interaction chromatography $x$ reversed phase liquid chromatography 424 hyphenated to high resolution mass spectrometry. I - Optimization of conditions J. 425 Chromatogr. B, 1032 (2016) 103-111.

426 [8] B.C. Laguzza, C.L. Nichols, S.L. Briggs, G.J. Cullinan, D.A. Johnson, J.J. Starling, New 427 antitumor monoclonal antibody-vinca conjugates LY203725 and related compounds: design, 428 preparation and representative in vivo activity. J Med Chem 32 (1989) 548-555.

429 [9] R.V. Chari, B.A. Martell, J.L. Gross, S.B. Cook, S.A. Shah, W.A. Blattler, 430 Immunoconjugates containing novel maytansinoids: promising anticancer drugs, Cancer Res $43152(1992) 27-31$. 
432 [10] F. Hudecz, M.C. Garnett, T. Khan, R.W. Baldwin, The influence of synthetic conditions 433 on the stability of methotrexate-monoclonal antibody conjugates determined by reversed 434 phase high performance liquid chromatography, Biomed Chromatogr 6 (1992) 128-132.

435 [11] R.V. Chari, K.A. Jackel, L.A. Bourret, S.M. Derr, B.M. Tadayoni, K.M. Mattocks, 436 Enhancement of the selectivity and antitumor efficacy of a CC-1065 analogue through 437 immunoconjugate formation, Cancer Res 55 (1995) 4079-4084.

438 [12] R.S. Greenfield, T. Kaneko, A. Daues, M.A. Edson, K.A. Fitzgerald, L.J. Olech, 439 Evaluation in vitro of adriamycin immunoconjugates synthesized using an acid-sensitive 440 hydrazone linker, Cancer Res 50 (1990) 6600-6607.

441 [13] D. Willner, P.A. Trail, S.J. Hofstead, H.D. King, S.J. Lasch, G.R. Braslawsky, (6442 Maleimidocaproyl)hydrazone of doxorubicin, a new derivative for the preparation of 443 immunoconjugates of doxorubicin, Bioconjug Chem 4 (1993) 521-527.

444 [14] M.M. Sun, K.S. Beam, C.G. Cerveny, K.J. Hamblett, R.S. Blackmore, M.Y. Torgov, 445 Reduction-alkylation strategies for the modification of specific monoclonal antibody 446 disulfides, Bioconjug Chem 16 (2005) 1282-1290.

447 [15] C.F. McDonagh, E. Turcott, L. Westendorf, J.B. Webster, S.C. Alley, K. Kim, Engineered 448 antibody-drug conjugates with defined sites and stoichiometries of drug attachment. Protein 449 Eng Des Sel 19 (2006) 299-307.

450 [16] K.J.Hamblett, P.D. Senter, D.F. Chace, M.M. Sun, J. Lenox, C.G. Cerveny, Effects of 451 drug loading on the antitumor activity of a monoclonal antibody drug conjugate, Clin Cancer 452 Res 10 (2004) 7063-7070.

453 [17] B. Bobaly, A. Beck, J.L. Veuthey, D. Guillarme, S. Fekete, Impact of organic modifier 454 and temperature on protein denaturationin hydrophobic interaction chromatography, J. 455 Pharm. Biomed. Anal, 131 (2016) 124-132.

456 [18] S. Fekete, J.L. Veuthey, A. Beck, D. Guillarme, Hydrophobic interaction chromatography 457 for the characterization of monoclonal antibodies and related products, J. Pharm. Biomed. 458 Anal, $130(2016)$ 3-18. 
459 [19] A. Cusumano, D. Guillarme, A. Beck, S. Fekete, Practical method development for the 460 separation of monoclonal antibodies and antibody-drug-conjugate species in hydrophobic 461 interaction chromatoraphy, part 2: Optimization of the phase system, J. Pharm. Biomed. 462 Anal, $121(2016)$ 161-173.

463 [20] M.M. Siegel, I.J. Hollander, P.R. Hamann, J.P. James, L. Hinman, B.J. Smith, Matrix464 assisted UV-laser desorption/ionization mass spectrometric analysis of monoclonal 465 antibodies for the determination of carbohydrate, conjugated chelator and conjugated drug 466 content, Anal Chem, 63 (1991) 2470-2481.

467 [21] J.R. Junutula, K.M. Flagella, R.A. Graham, K.L Parsons, E. Ha, H. Raab, Engineered 468 thio-trastuzumab-DM1 conjugate with an improved therapeutic index to target human 469 epidermal growth factor receptor 2-positive breast cancer, Clin Cancer Res 16 (2010) 47694704778.

471 [22] L. Wang, G. Amphlett, W.A. Blattler, J.M. Lambert, W. Zhang, Structural characterization 472 of the maytansinoid-monoclonal antibody immunoconjugate, huN901-DM1, by mass 473 spectrometry, Protein Sci 14 (2005) 2436-2446.

474 [23] A.C. Lazar, L. Wang, W.A. Blattler, G. Amphlett, J.M. Lambert, W. Zhang, Analysis of 475 the composition of immunoconjugates using size-exclusion chromatography coupled to mass 476 spectrometry, Rapid Commun Mass Spectrom, 19 (2005) 1806-1814.

477 [24] M.C.Janin Bussat, M. Dillenbourg, N. Corvaia, A. Beck, C. Klinguer-Hamour, 478 Characterization of antibody drug conjugate positional isomers at cysteine residues by 479 peptide mapping LC-MS analysis, J. Chromatogr. B, 981-982 (2015) 9-13.

480 [25] T. Chen, Y. Chen, C. Stella, C.D. Medley, J.A. Gruenhagen, K. Zhang, Antibody-drug 481 conjugate characterization by chromatographic andelectrophoretic techniques, J. 482 Chromatogr. B, 1032 (2016) 39-50.

483 [26] S. Fekete, S. Rudaz, J. Fekete, D. Guillarme, Analysis of recombinant monoclonal 484 antibodies by RPLC: towards a generic method development approach, J. Pharm. Biomed. 485 Anal., 70 (2012) 158-168. 
486 [27] S. Fekete, S. Rudaz, J.L. Veuthey, D. Guillarme, Impact of mobile phase temperature on 487 recovery and stability of monoclonal antibodies using recent reversed-phase stationary 488 phases, J. Sep. Sci., 35 (2012) 3113-3123.

489 [28] J.A. Lewis, L.R. Snyder, J.W. Dolan, Initial experiments in high-performance liquid 490 chromatographic method development II. Recommended approach and conditions for 491 isocratic separation, J. Chromatogr. A 721 (1996) 15-29.

492 [29] J.W. Dolan, L.R. Snyder, N.M. Djordjevic, D.W. Hill, D.L. Saunders, L. Van Heukelem, 493 T.J. Waeghe, Simultaneous variation of temperature and gradient steepness for reversed494 phase high-performance liquid chromatography method development: I. Application to 14 495 different samples using computer simulation, J. Chromatogr. A 803 (1998) 1-31.

496 [30] L.R. Snyder, J.J. Kirkland, J.L. Glajch, Practical HPLC Method Development, second 497 ed., John Wiley \& Sons Inc., 1997.

498 [31] J.C. Berridge, Techniques for the Automated Optimization of HPLC Separations, John 499 Wiley \& Sons, New York, 1986.

500 [32] R. Kormány, I. Molnár, H.J. Rieger, Exploring better column selectivity choices in ultra501 high performance liquid chromatography using Quality by Design principles, J. Pharm. 502 Biomed. Anal., 80 (2013) 79-88.

503 [33] N. Rácz, R. Kormány, J. Fekete, I. Molnár, Establishing column batch repeatability 504 according to Qualityby Design (QbD) principles using modeling software, J. Pharm. Biomed. 505 Anal., $108(2015)$ 1-10.

506 [34] R. Kormány, J. Fekete, D. Guillarme, S. Fekete, Reliability of simulated robustness 507 testing in fast liquid chromatography, using state-of-the-art column technology, 508 instrumentation and modelling software, J. Pharm. Biomed. Anal., 89 (2014) 67-75.

509 [35] Y. Chen, C.T. Mant, R.S. Hodges, Temperature selectivity effects in reversed-phase 510 liquid chromatography due to conformation differences between helical and non-helical 511 peptides, J. Chromatogr. A 1010 (2003) 45-61. 
512 [36] C.T. Mant, Y. Chen, R.S. Hodges, Temperature profiling of polypeptides in reversed-

513 phase liquid chromatography: I. Monitoring of dimerization and unfolding of amphipathic $\alpha-$

514 helical peptides, J. Chromatogr. A 1009 (2003) 29-43.

515 [37] C.T. Mant, B. Tripet, R.S. Hodges, Temperature profiling of polypeptides in reversed-

516 phase liquid chromatography: II. Monitoring of folding and stability of two-stranded $\alpha$-helical

517 coiled-coils, J. Chromatogr. A 1009 (2003) 45-59.

518 [38] S. Fekete, A. Beck, E. Wagner, K. Vuignier, D. Guillarme, Adsorption and recovery

519 issues of recombinant monoclonal antibodies in reversed-phase liquid chromatography, J.

520 Sep. Sci., 38 (2015) 1-8.

521 [39] A.H. Schmidt, I. Molnár, Using an innovative Quality-by-Design approach for

522 development of a stability indicating UHPLC method for ebastine in the API and

523 pharmaceutical formulations, J. Pharm. Biomed. Anal., 78-79 (2013) 65-74.

524 
Figure captions

527 Figure 1. The impact of gradient steepness on selectivity. Column: Agilent Advance BioMAb RP C4. Mobile phase "A": 0.1\% TFA in water, "B": 0.1\% TFA in acetonitrile. Flow rate: 0.3 $\mathrm{mL} / \mathrm{min}$, gradient: $25-50 \% \mathrm{~B}$ (in $6 \min (\mathrm{A}), 12 \mathrm{~min}(\mathrm{~B})$ and $18 \mathrm{~min}(\mathrm{C})$ ), temperature: $90{ }^{\circ} \mathrm{C}$,

530 injected volume: $1 \mu \mathrm{L}$, detection at $280 \mathrm{~nm}$. Panel $\mathrm{D}$ shows the dependence of $k_{\text {app }}$ on the

531 gradient time. Sample: Brentuximab vedotin

Figure 2. The impact of mobile phase temperature on the retention and peak area (recovery) of different ADC species. Column: Agilent Advance BioMAb RP C4. Mobile phase "A": 0.1\% TFA in water, "B": $0.1 \%$ TFA in acetonitrile. Flow rate: $0.3 \mathrm{~mL} / \mathrm{min}$, gradient: $30-60 \% \mathrm{~B}$ in 6 min (extended gradient to enable baseline resolution and the measurement of peak areas),

537 temperature: $90{ }^{\circ} \mathrm{C}$, injected volume: $1 \mu \mathrm{L}$, detection at $280 \mathrm{~nm}$. Sample: Brentuximab

538 vedotin

Figure 3. The impact of mobile phase ternary composition. Column: Agilent Advance BioMAb RP C4. Mobile phase "A": 0.1\% TFA in water, "B": 0.1\% TFA in acetonitrile and methanol mixture. Flow rate: $0.3 \mathrm{~mL} / \mathrm{min}$, gradient: $30-80 \% \mathrm{~B}$ in 6 min (extended gradient to enable

543 eluting all the peaks with $50 \% \mathrm{MeOH}$ content), temperature: $90{ }^{\circ} \mathrm{C}$, injected volume: $1 \mu \mathrm{L}$,

544 detection at $280 \mathrm{~nm}$. Sample: Brentuximab vedotin

Figure 4. Suggested experimental design for 3D retention model (column: $150 \times 2.1 \mathrm{~mm}$, gradient: $25-50 \%$ B at $0.3 \mathrm{~mL} / \mathrm{min}$ ).

549 Figure 5. Model verification in the center point for native Brentuximab vedotin (1) and for 550 reduced Brentuximab vedotin (2). Column: Agilent Advance BioMAb RP C4. Mobile phase 551 "A": $0.1 \%$ TFA in water, "B": $0.1 \%$ TFA in 90\% acetonitrile $+10 \% \mathrm{MeOH}$. Flow rate: 0.3 $552 \mathrm{~mL} / \mathrm{min}$, gradient: $25-50 \% \mathrm{~B}$ in $15 \mathrm{~min}$, temperature: $82.5{ }^{\circ} \mathrm{C}$, injected volume: $1 \mu \mathrm{L}$, 
553 detection at $280 \mathrm{~nm}$. "A" corresponds to predicted, while "B" corresponds to experimental

554 chromatograms.

555

556 Figure 6. 3D resolution maps for Brentuximab vadotin $(A)$ and for Brentuximab vedotin

557 reduced $(B)$ samples, based on the initial experiments (Rs crit $=1.0$ ). Set conditions for the

558 native sample: $t_{G}=23 \mathrm{~min}, \mathrm{~T}=88^{\circ} \mathrm{C}$ and $t C=5 \% \mathrm{MeOH}$. Set conditions for the reduced

559 sample: $t_{G}=27 \mathrm{~min}, \mathrm{~T}=87^{\circ} \mathrm{C}$ and $t C=5 \% \mathrm{MeOH}$.

560

561 Figure 7. Predicted (A) and experimentally verified (B) chromatograms of native Brentuximab 562 vedotin under optimal conditions. Gradient: $29-42 \% \mathrm{~B}, t_{G}=14 \mathrm{~min}, \mathrm{~T}=90{ }^{\circ} \mathrm{C}$ and $t \mathrm{C}=0 \%$ $563 \mathrm{MeOH}(100 \% \mathrm{AcN})$. (Column: Agilent Advance BioMAb RP C4, flow rate: $0.3 \mathrm{~mL} / \mathrm{min}$ )

564

565 Figure 8. Predicted (A) and experimentally verified (B) chromatograms of reduced 566 Brentuximab vedotin under optimal conditions to optimize resolution between L1 and H0 567 species. Gradient: $31-48 \% \mathrm{~B}, t_{G}=18 \mathrm{~min}, \mathrm{~T}=90{ }^{\circ} \mathrm{C}$ and $t \mathrm{C}=20 \% \mathrm{MeOH}(80 \% \mathrm{AcN})$. 568 (Column: Agilent Advance BioMAb RP C4, flow rate: $0.3 \mathrm{~mL} / \mathrm{min}$ )

570 Figure 9. Predicted (A) and experimentally verified (B) chromatograms of reduced

571 Brentuximab vedotin under optimal conditions to optimize resolution between all peaks.

572 Gradient: $25-50 \% \mathrm{~B}, t_{G}=27 \mathrm{~min}, \mathrm{~T}=87^{\circ} \mathrm{C}$ and $t \mathrm{C}=5 \% \mathrm{MeOH}(95 \% \mathrm{AcN})$. (Column: 573 Agilent Advance BioMAb RP C4, flow rate: $0.3 \mathrm{~mL} / \mathrm{min}$ ). Panel C represents the result of 574 simulated robustness test as number of observations $(N)$ vs. critical resolution $\left(R_{s, \text { crit }}\right)$.

575

576 Figure 10. Simplified 2D experimental designs as $t_{G} \times \mathrm{T}$ model (4 runs) and $t_{G} \times t C$ (6 runs) 577 models 
579 Figure 11. Simplified 2D resolution maps based on four initial experiments ( $t_{G} \times T$ model). 580 Gradient: $25-50 \% \mathrm{~B}, t_{G 1}=10 \mathrm{~min}, t_{\mathrm{G} 2}=20 \mathrm{~min}, \mathrm{~T}_{1}=75^{\circ} \mathrm{C}, \mathrm{T}_{2}=90^{\circ} \mathrm{C}$ and $t \mathrm{C}=0 \% \mathrm{MeOH}$ 581 (100\% AcN). (Column: Agilent Advance BioMAb RP C4, flow rate: $0.3 \mathrm{~mL} / \mathrm{min}$ ) 582

583 Figure 12. Simplified 2D resolution maps based on six initial experiments $\left(t_{G} \times t C\right.$ model).

584 Gradient: $25-50 \% \mathrm{~B}, t_{G 1}=10 \mathrm{~min}, t_{G 2}=20 \mathrm{~min}, t C_{1}=0 \% \mathrm{MeOH}, t C_{2}=10 \% \mathrm{MeOH}$ and $t C_{3}$ $585=20 \% \mathrm{MeOH}, \mathrm{T}=90^{\circ} \mathrm{C}$. (Column: Agilent Advance BioMAb RP C4, flow rate: $0.3 \mathrm{~mL} / \mathrm{min}$ ) 586 
587 Tables

588

589 Table 1. Prediction accuracy in the center point of the experimental design for native

590 brentuximab vedotin

591

592 Table 2. Prediction accuracy in the center point of the experimental design for

593 reduced brentuximab vedotin

594 

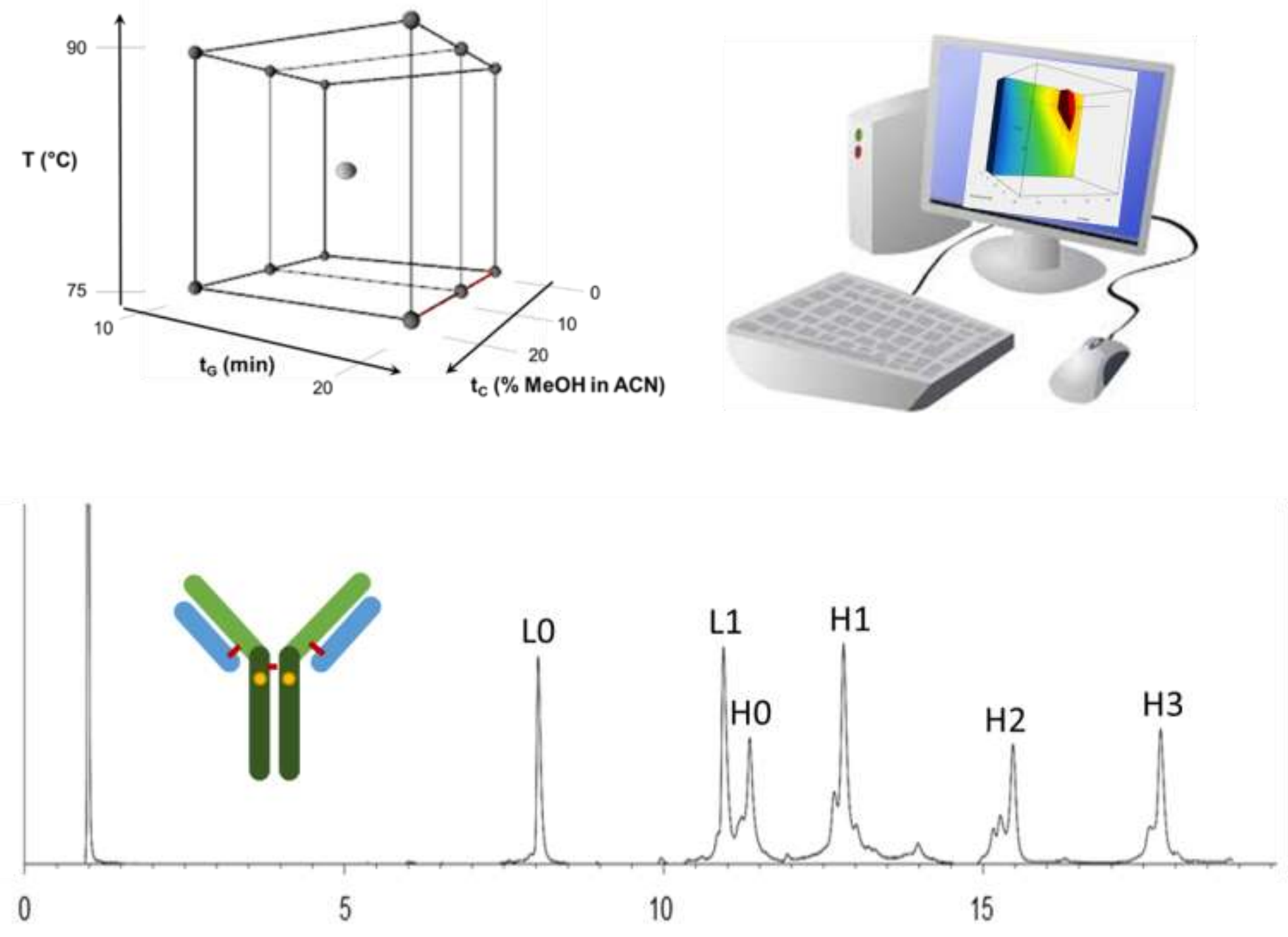

retention time (min) 

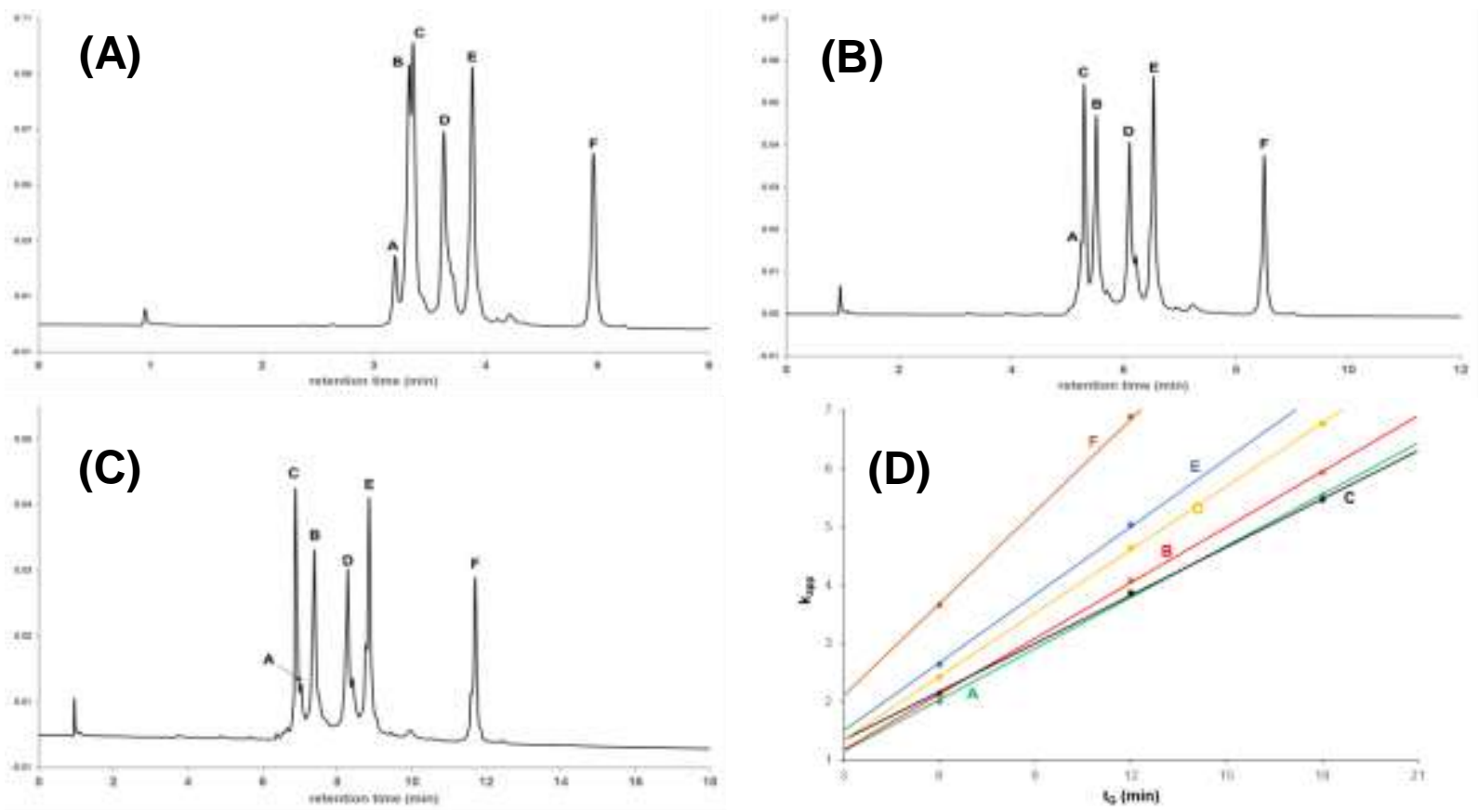

Figure 1. 

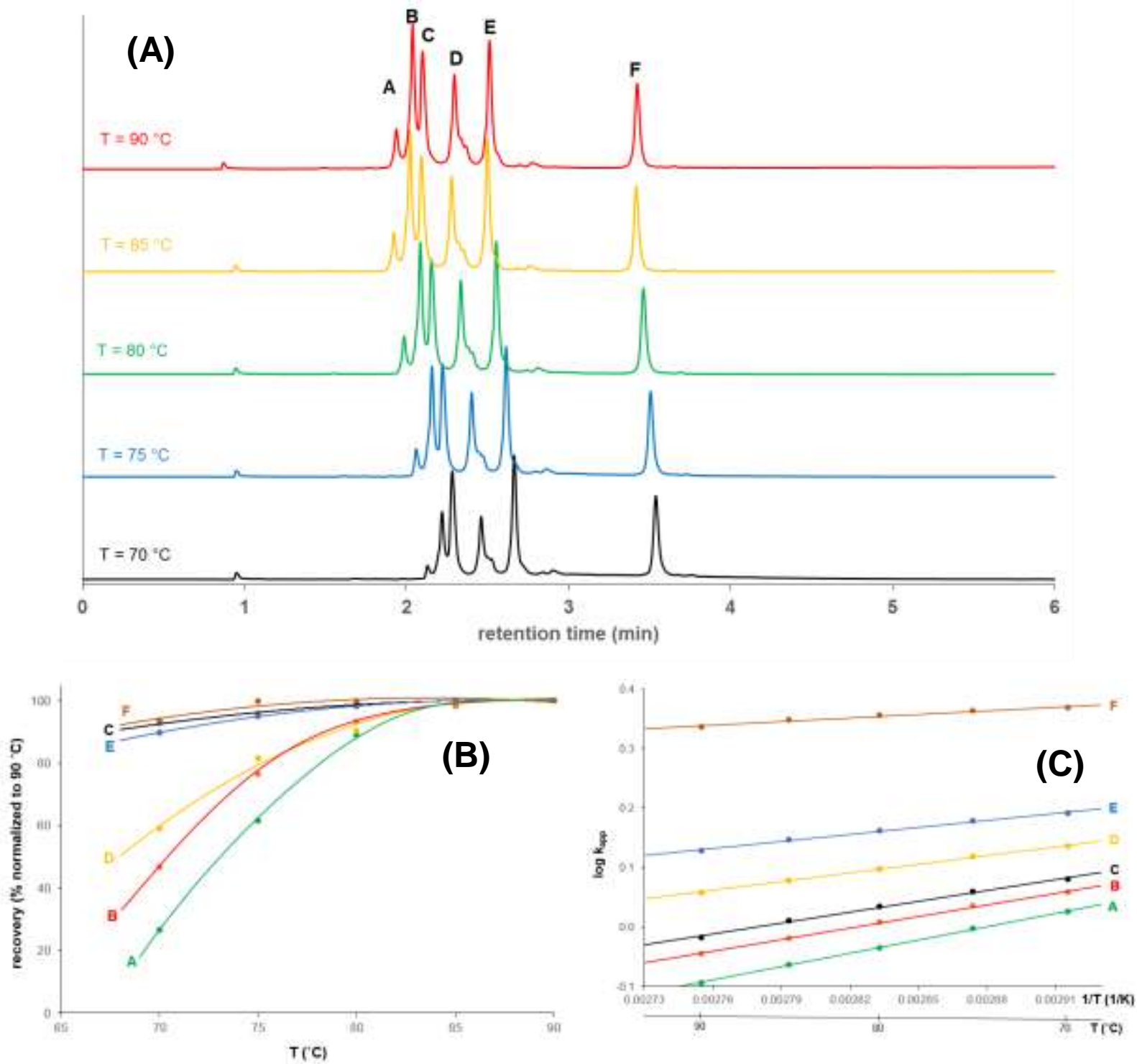

Figure 2. 


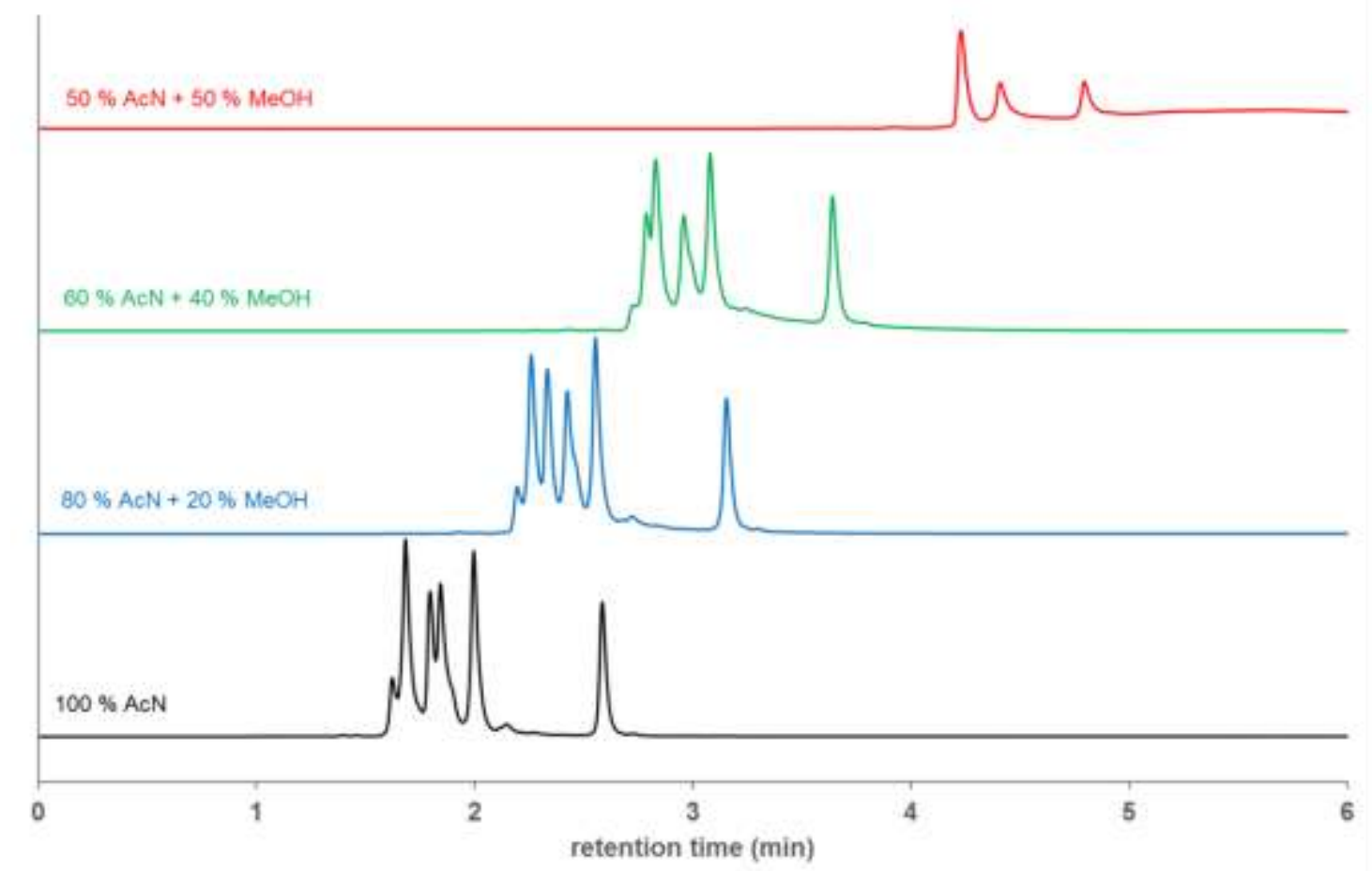

Figure 3. 


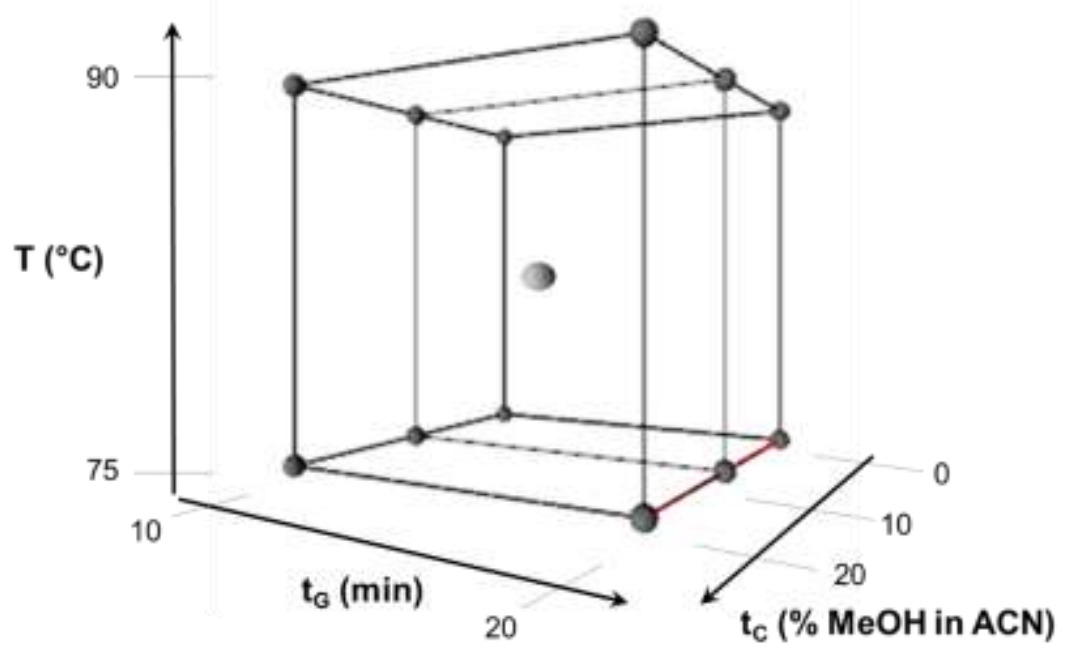

Figure 4. 

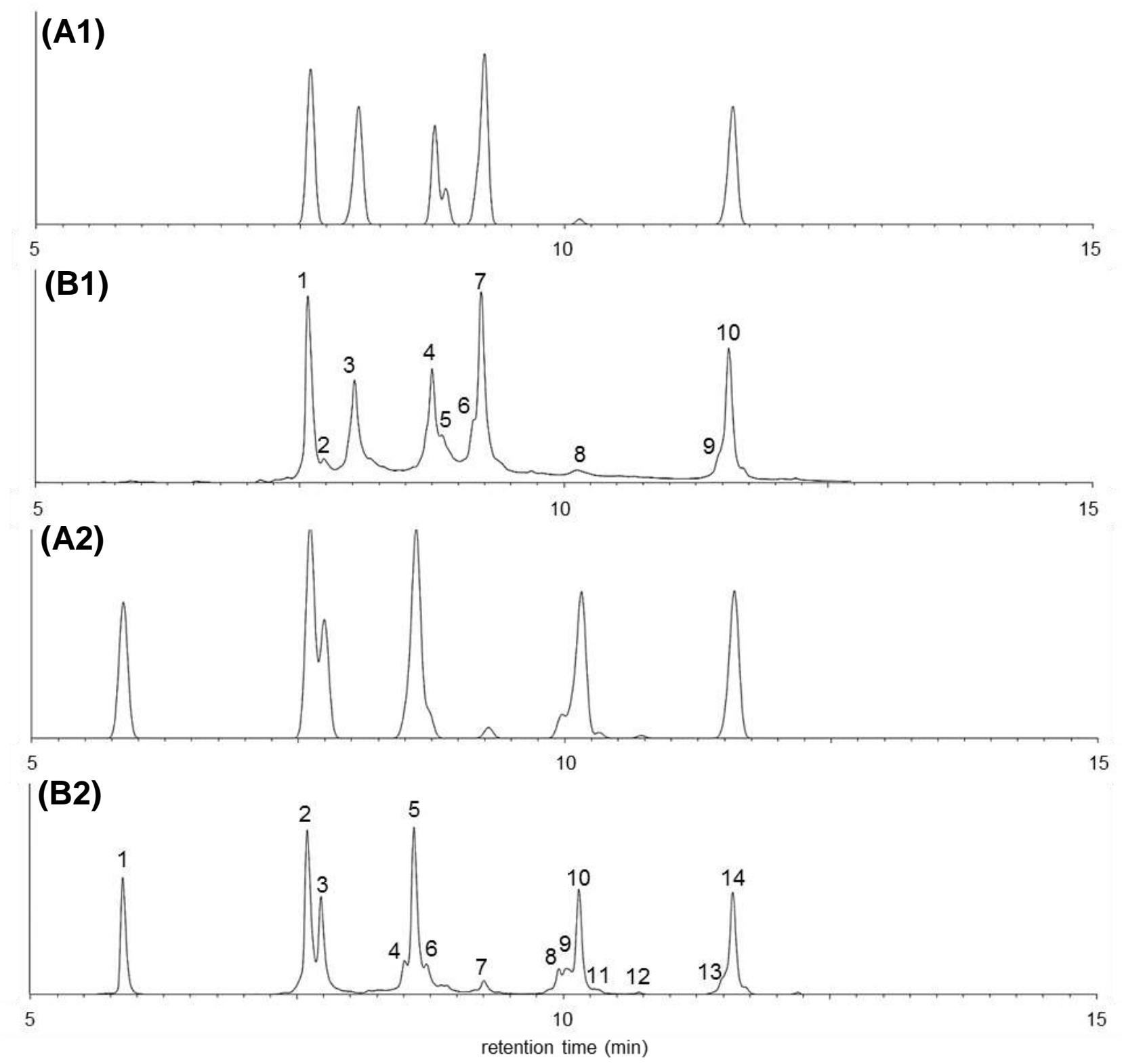

Figure 5. 

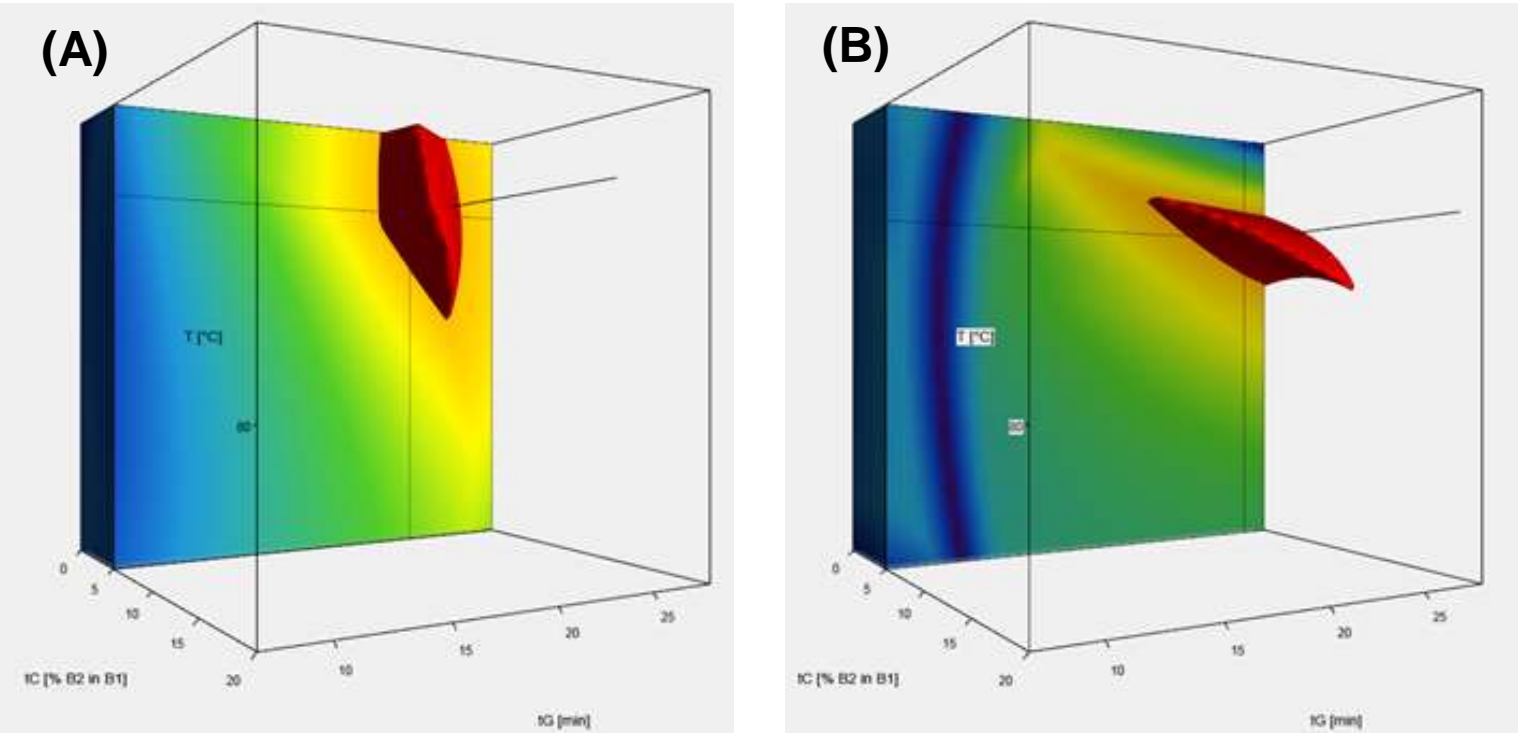

Figure 6. 
(A)

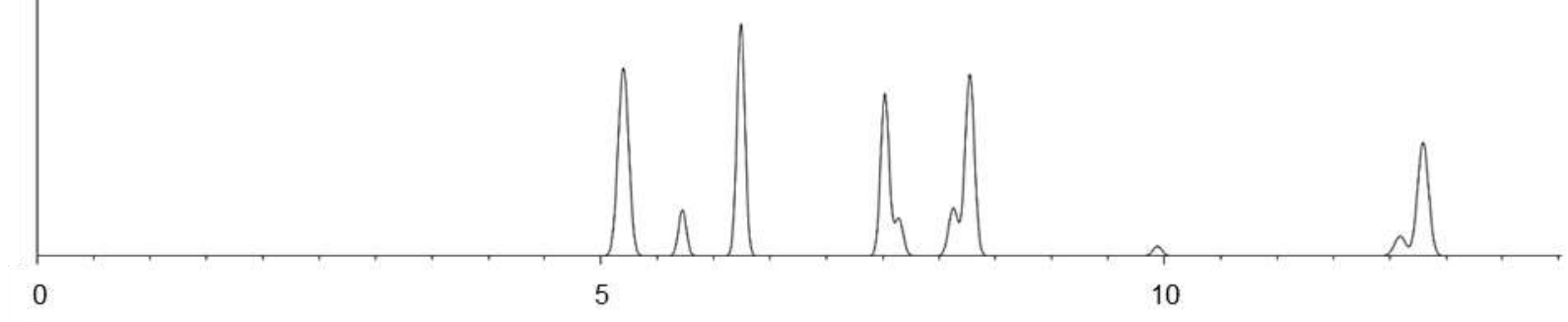

(B)

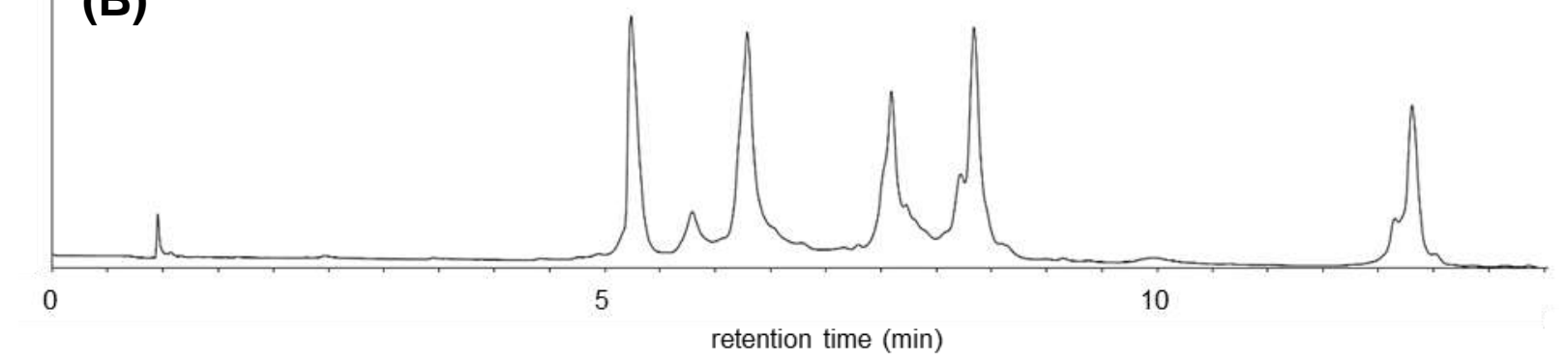

Figure 7. 
(A)

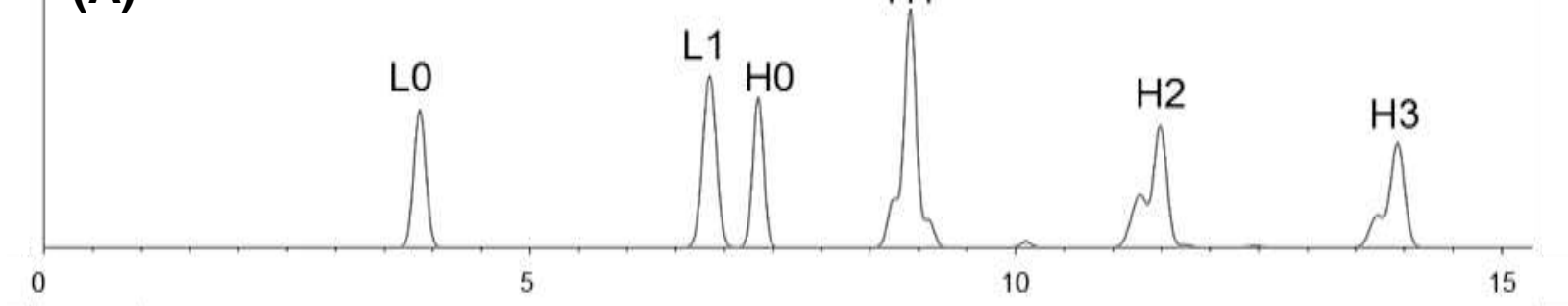

(B)

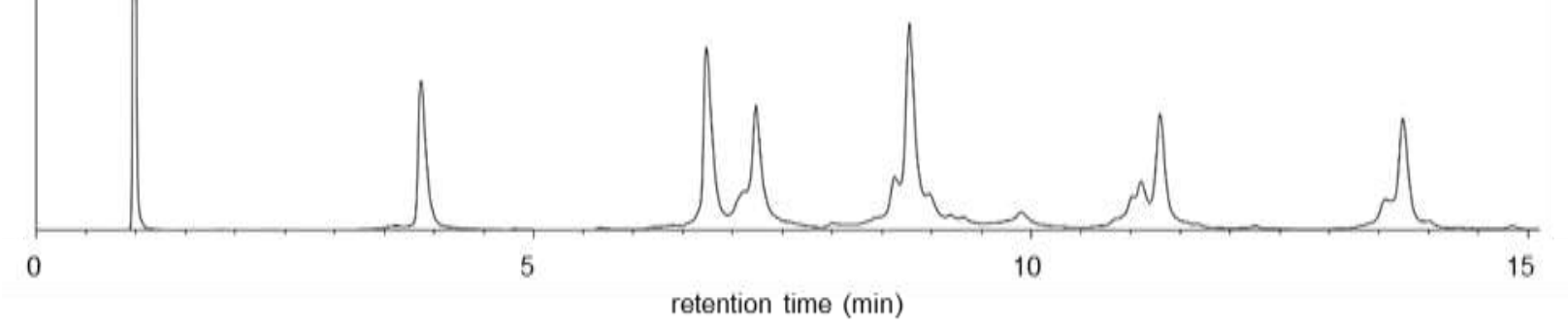

Figure 8. 

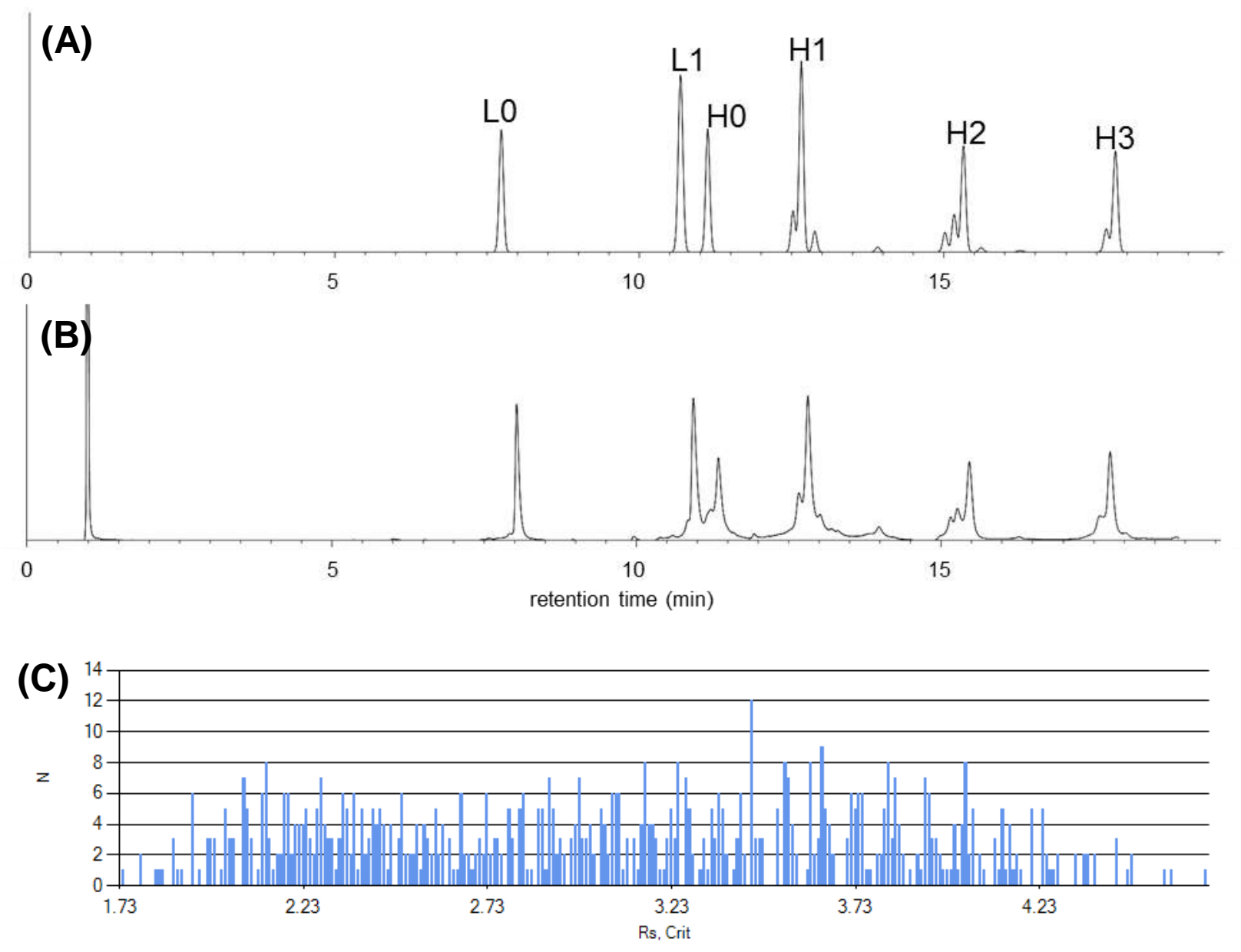

Figure 9. 

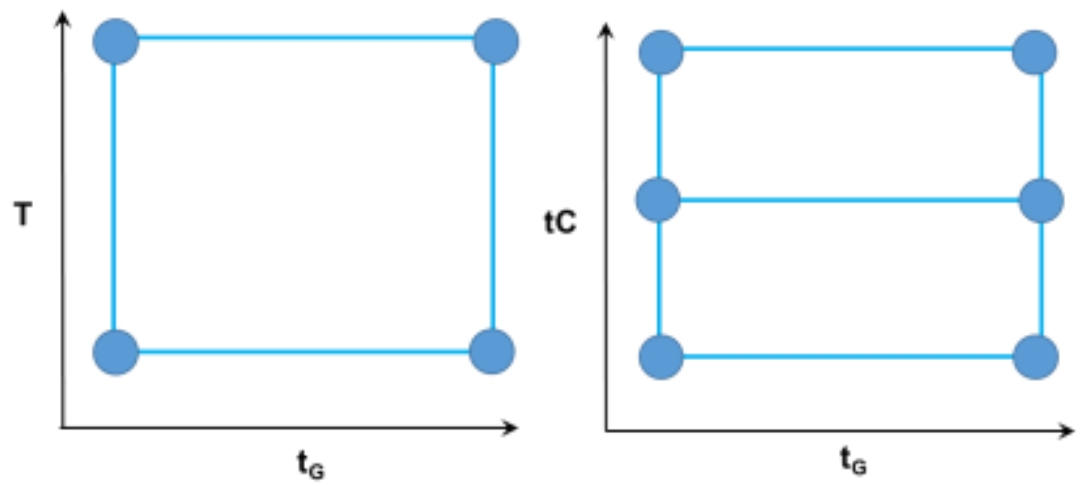

Figure 10. 

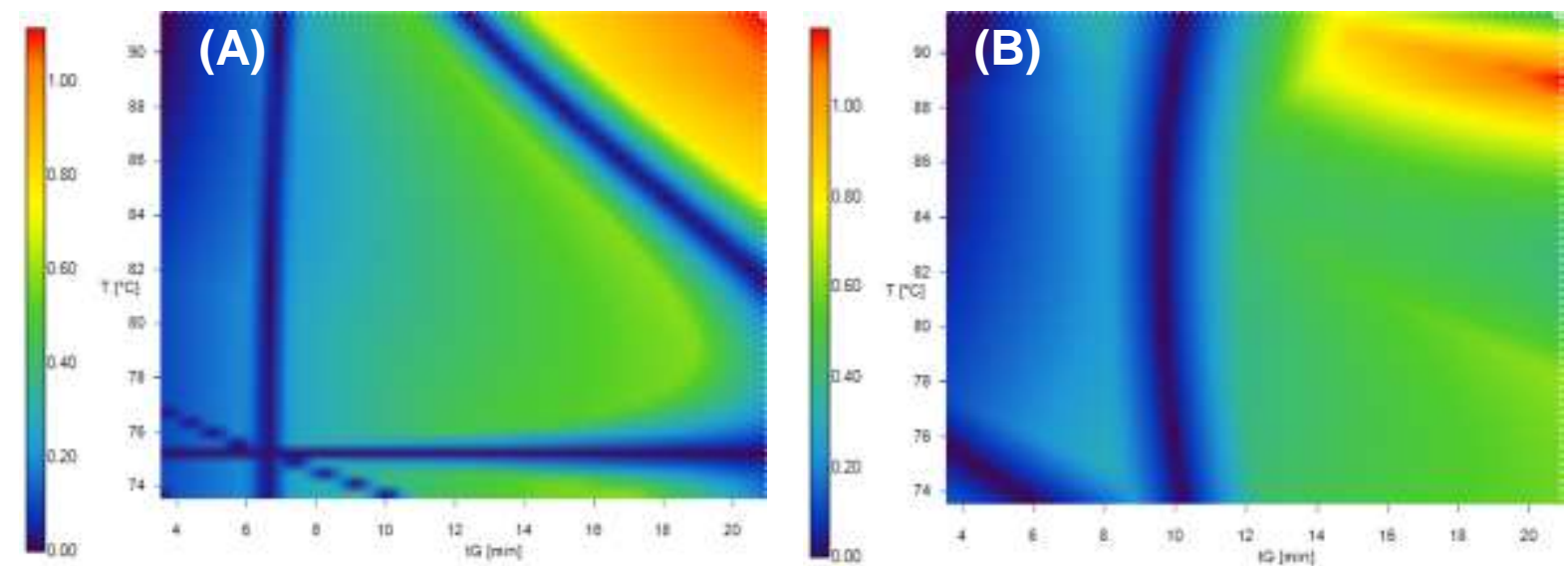

Figure 11. 

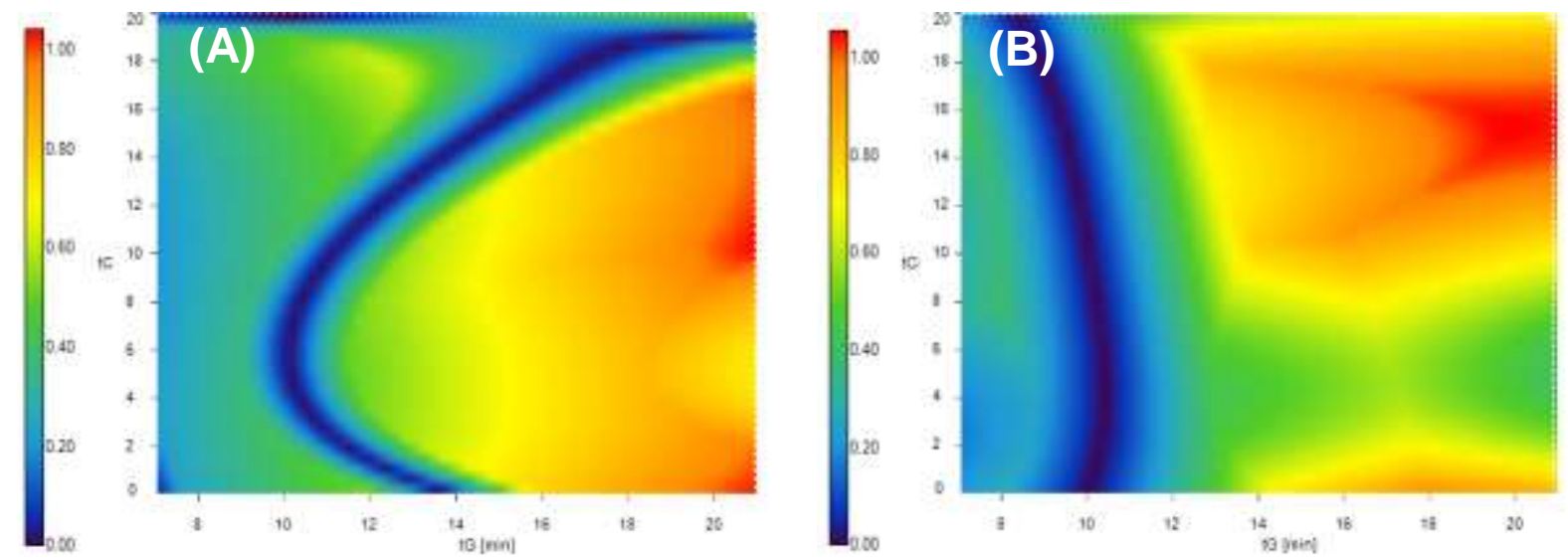

Figure 12. 
Table 1.

\begin{tabular}{ccccc} 
& $\mathrm{t}_{\mathrm{r}}$ experimental $(\mathrm{min})$ & $\mathrm{t}_{\mathrm{r}}$ predicted $(\mathrm{min})$ & Difference $^{\mathrm{a}}$ & \% error $^{\mathrm{b}}$ \\
\hline peak 1 & 7.575 & 7.590 & -0.01 & -0.20 \\
peak 2 & 7.726 & 7.740 & -0.01 & -0.18 \\
peak 3 & 8.016 & 8.050 & -0.03 & -0.42 \\
peak 4 & 8.749 & 8.770 & -0.02 & -0.24 \\
peak 5 & 8.844 & 8.870 & -0.03 & -0.29 \\
peak 6 & 9.149 & 9.170 & -0.02 & -0.23 \\
peak 7 & 9.215 & 9.240 & -0.03 & -0.27 \\
peak 8 & 10.124 & 10.140 & -0.02 & -0.16 \\
peak 9 & 11.507 & 11.530 & -0.02 & -0.20 \\
peak 10 & 11.557 & 11.590 & -0.03 & -0.28 \\
& & average & -0.02 & -0.25
\end{tabular}

${ }^{\mathrm{a}}$ Difference $=$ experimental-predicted

${ }^{\mathrm{b}} \%$ error $=[($ experimental - predicted $) /$ predicted $] \times 100$ 
Table 2.

\begin{tabular}{ccccc} 
& $\mathrm{t}_{\mathrm{r}}$ experimental $(\mathrm{min})$ & $\mathrm{t}_{\mathrm{r}}$ predicted $(\mathrm{min})$ & Difference $^{\mathrm{a}}$ & ${\text { \% } \text { error }^{\mathrm{b}}}$ \\
\hline peak 1 & 5.868 & 5.850 & 0.02 & 0.31 \\
peak 2 & 7.599 & 7.610 & -0.01 & -0.14 \\
peak 3 & 7.729 & 7.740 & -0.01 & -0.14 \\
peak 4 & 8.514 & 8.520 & -0.01 & -0.07 \\
peak 5 & 8.601 & 8.600 & 0.00 & 0.01 \\
peak 6 & 8.718 & 8.720 & 0.00 & -0.02 \\
peak 7 & 9.258 & 9.280 & -0.02 & -0.24 \\
peak 8 & 9.961 & 9.960 & 0.00 & 0.01 \\
peak 9 & 10.031 & 10.070 & -0.04 & -0.39 \\
peak 10 & 10.148 & 10.150 & 0.00 & -0.02 \\
peak 11 & 10.297 & 10.320 & -0.02 & -0.22 \\
peak 12 & 10.711 & 10.710 & 0.00 & 0.01 \\
peak 13 & 11.543 & 11.530 & 0.01 & 0.11 \\
peak 14 & 11.592 & 11.590 & 0.00 & 0.02 \\
& & average & -0.01 & -0.06
\end{tabular}

\footnotetext{
${ }^{a}$ Difference $=$ experimental-predicted

${ }^{b} \%$ error $=[($ experimental - predicted $) /$ predicted $] \times 100$
} 